\title{
El liderazgo vietnamita después del XII Congreso del Partido Comunista de Vietnam y la continuidad de las reformas económicas*
}

\section{Vietnamese leadership after the XIlth Congress of the Communist Party of Vietnam and the continuity of economic reforms}

\author{
Mauricio de Miranda* \\ Pavel Vidal**
}

Fecha de recepción: 23 de junio de 2017 Fecha de aprobación: 13 de octubre de 2017

\section{Resumen}

El presente artículo analiza los cambios en el liderazgo político vietnamita a partir de la celebración del $12^{\circ}$ Congreso del Partido Comunista Vietnamita (PCV). Estos cambios son definitorios para la continuidad de la reforma y la inserción internacional

* El documento es el resultado del trabajo de investigación desarrollado por los autores en una serie de proyectos apoyados por la Pontificia Universidad Javeriana Cali sobre Asia y Pacífico, entre los que se encuentra: Análisis del modelo de Crecimiento Económico con Restricción de Balanza de Pagos (CRBP) en Colombia desde las perspectivas de las relaciones económicas con Asia. Un especial agradecimiento a José Tomás Pelaez y Yined Marcela Lopez por su importante ayuda en el desarrollo de la investigación.

* Doctor en Economía Internacional y Desarrollo, Universidad Complutense de Madrid. Profesor Titular y Director del Centro de Estudios sobre la Cuenca del Pacífico de la Pontificia Universidad Javeriana Cali. Correo: mdmirand@javerianacali.edu.co

** Doctor en Economía de la Universidad de La Habana. Profesor Asociado del Departamento de Economía de la Pontificia Universidad Javeriana Cali. Correo: pavel@javerianacali.edu.co 
de Vietnam, y son claves que requieren conocer los empresarios y funcionarios colombianos para poder penetrar un mercado que por el momento no ha sido aprovechado al máximo. En el nuevo Buró Político vietnamita fueron ratificados sólo siete de los 16 miembros del anterior equipo. Se ha fortalecido, al menos temporalmente, la posición del Secretario General Nguyen Phu Trong, lo que, junto a otras designaciones, otorga un leve giro hacia una tendencia "conservadora". Pero ello no implica que se vayan a revertir las reformas, sino más bien que se reforzará el control político e ideológico del PCV.

\section{Palabras clave}

Vietnam, Partido Comunista, Liderazgo, Reforma

\section{Abstract}

This article analyzes the changes in Vietnamese political leadership from the celebration of the 12th Congress of the Vietnamese Communist Party (PCV). These changes are crucial for the continuity of the reform and the international insertion of Vietnam, and they are keys that need to know the Colombian businessmen and officials to be able to penetrate a market that for the moment has not been exploited to the maximum. In the new Vietnamese Political Bureau only seven of the 16 members of the previous team were ratified. The position of Secretary-General Nguyen Phu Trong has been strengthened, at least temporarily, which gives a slight twist to a "conservative" trend. But this does not mean that reforms will be reversed, but rather that the political and ideological control of the PCV will be strengthened.

\section{Keywords}

Vietnam, Communist Party, Leadership, Reform 


\section{Introducción}

El intercambio comercial de Colombia con Asia ha tenido una tendencia creciente desde el comienzo del presente siglo. La contribución de Asia a la expansión del PIB colombiano se estima en $0,6 \%$, de una tasa de crecimiento promedio del PIB de 4,1\%. En particular China, pasó a ser, el socio comercial con la segunda mayor contribución, por debajo solo de EEUU, y por encima de socios tradicionales como Ecuador, Perú y Venezuela (Vidal, de Miranda y Peláez, 2013). ${ }^{1}$

Sin embargo, desde el año 2012 la economía china viene transitando por un proceso de desaceleración debido a que las autoridades vienen reestructurando su modelo de crecimiento apoyado en exportaciones, inversiones y endeudamiento de las empresas públicas, a uno impulsado en el consumo, la expansión de la clase media y la liberalización de la cuenta financiera de la balanza de pagos. Vidal y Rodriguez (2016) estiman que como acumulado, de 2012-2015, la desaceleración en China ha tenido ya un efecto negativo que equivale al $0,73 \%$ sobre el crecimiento del PIB colombiano.

Ante la continuada desaceleración de la economía china, parece estratégico explorar otros mercados dentro de la región más dinámica del mundo en términos de crecimiento y comercio internacional, la cual sigue siendo Asia. La economía de Vietnam es una de las opciones a valorar, dado su potencial y resilencia a las tendencias al estancamiento y mayor volatilidad que experimenta la economía mundial.

El comercio bilateral entre Colombia y Vietnam se ha dinamizado en la última década, sobre todo debido al incremento de las

1 En un estudio del SELA (2016) se encuetra que el MERCOSUR concentra gran parte del comercio con la ASEAN al acaparar el $83 \%$ de las exportaciones en bienes primarios a esta región. 
importaciones colombianas desde Vietnam. ${ }^{2}$ Las cifras apuntan a que Colombia no ha sabido aprovechar la dinámica expansiva de la economía vietnamita. Son extremadamente bajos los valores exportados y creciente el déficit qua viene acumulando Colombia en su comercio con el país asiático. El desconocimiento de las reformas y oportunidades que el gobierno vietnamita ofrece a sus contrapartes, puede ser uno de los factores que influyen en este resultado tan poco satisfactorio para el comercio colombiano. Es por ello, que entender las lógicas de las dinámicas políticas resulta en un elemento crucial, pues es un país con un sistema de partido único, cuyos códigos es necesario entender para poder penetrar un mercado donde lo económico y lo político tienen una relación más directa, que en economías con democracias liberales.

Vietnam emprendió una reforma profunda de su sistema económico desde finales de los años ochenta. La reforma ha sido efectiva en promover una tasa de expansión de la economía, la reducción del índice de pobreza de $60 \%$ a $10 \%$ y una inserción acelerada en los mercados internacionales (Chu and Dickie, 2006). La liberalización de los mercados domésticos, la apertura a la inversión extranjera directa, la reforma del sistema empresarial estatal y el regreso a las instituciones financieras multilaterales, han sido cruciales en sus dinámica positivas de progreso económico. Vietnam pasó de ser una economía

2 De acuerdo con los datos de Trademap (www.trademap.org), Colombia exportó hacia Vietnam tan solo un valor promedio de 5 millones de dólares en los últimos quince años, registrando su valor máximo de 18 millones de dólares en 2013. Mientras que las compras colombianas a Vietnam contaron con un valor promedio de 130 millones de dólares. A partir del año 2011 las importaciones desde Vietnam superaron la línea de los 100 millones de dólares. El déficit comercial bilateral pasó de 5 millones de dólares en 2001 a 482 millones de dólares en 2015. El comercio entre Colombia y Vietnam no presenta una alta diversificación. Alrededor del $50 \%$ de las exportaciones de Colombia hacia Vietnam están concentradas en tan solo tres productos. En 2015 el primer producto de exportación eran las pieles y cueros, seguido de tabaco, y madera y carbón vegetal. Mientras que los productos importados por Colombia desde Vietnam presentan una concentración aún mayor, dado que tan solo tres productos superan más del $60 \%$ del total. El calzado, polainas y artículos análogos tenía una participación del $52 \%$ para el 2001, del $25 \%$ para el 2007 y del $16 \%$ para el 2015. En 2015 el primer producto de importación lo ocupan las máquinas, aparatos y materiales eléctricos. 
básicamente agrícola a una dominada por la manufactura y el sector de bienes raíces. La tasa de apertura económica pasó de $50 \%$ a inicio de los años noventa a más del $100 \%$ del PIB en los años dos mil. En el mismo período la tasa de inversión aumentó de $18 \%$ a más del $30 \%$ del PIB. El Producto Interior Bruto (PIB) per cápita, a precios constantes de 2005, se incrementó de 237 dólares estadounidenses (USD) en 1986 a 1.003 en 2014, mientras que la tasa de crecimiento promedio anual del PIB ha sido de 6,6\% y la del PIB per cápita, 5,1\%, durante el período mencionado (UNCTAD, 2015).

Sin embargo, tal camino de progreso económico e inserción global no ha sido lineal, sino que ha estado marcado por numerosos desafíos institucionales, contradicciones en los mandos políticos y resistencias a los cambios. Si bien el mercado y la propiedad privada dominan el sistema económico, todavía hay una alta presencia e intervención del Estado en la economía y se mantiene el control sobre los flujos de capitales y el sistema bancario, todo ello dentro de un modelo que se ha dado en llamar: economía socialista de mercado. En este camino, el Partido Comunista y su liderazgo han definido las pautas de las transformaciones.

Los congresos de los Partidos Comunistas en los países en los que éstos gobiernan bajo un régimen de partido único suelen ser acontecimientos fundamentales en la vida política de los mismos, puesto que en ellos se realiza un balance de la actividad del partido, así como del contexto nacional e internacional del período previo, se trazan las políticas fundamentales para el período siguiente, usualmente de cinco años y se elige al nuevo liderazgo, encabezado por el Secretario General, el Buró Político, el Secretariado y el Comité Central. Y en ciertas ocasiones, puntuales, incluso pueden definirse estrategias de desarrollo de más largo plazo.

Entre el 21 y el 28 de enero de 2016 sesionó el $12^{\circ}$ Congreso del Partido Comunista de Vietnam (PCV), siguiendo el formato 
tradicional que venía desde los congresos del Partido Comunista de la Unión Soviética, y de los demás países del sistema socialista mundial.

Este Congreso, al parecer, había generado mucha expectativa porque se esperaba una renovación considerable del liderazgo y se había apostado incluso a la posibilidad del reemplazo del secretario general, Nguyen Phu Trong, (71 años). Algunos medios de prensa y expertos consideraban que el más firme candidato para dicho puesto era el saliente Primer Ministro Nguyen Tan Dung (66 años), quien en mayo de 2016 terminaría sus dos períodos posibles de cinco años en la jefatura del gobierno y, aparentemente, era el dirigente más poderoso del equipo dirigente de Vietnam. ${ }^{3}$ Otros analistas, mencionaban a su rival, el Presidente Truong Tan Sang (67). Sin embargo, las cábalas no resultaron. Trong fue reelegido secretario general para un segundo mandato y Dung y Sang quedaron por fuera del Buró Político e incluso del Comité Central. Mientras tanto, en el nuevo Buró Político, de sus 19 miembros, 12 son nuevos y sólo siete han sido reelegidos del anterior congreso. En el nuevo organismo han sido excluidos cuatro de los cinco principales cargos del país, pues además del Presidente y al Primer Ministro, se excluyó al Presidente de la Asamblea Nacional, Nguyen Sinh Hung, y al, hasta entonces, secretario ejecutivo del Comité Central, Le Hong Anh, que hace las veces de segundo secretario del Partido.

El presente artículo analiza los cambios en el liderazgo político vietnamita a partir de la celebración del $12^{\circ}$ Congreso del PCV, con el objeto de analizar la posible dirección del proceso de reformas económicas, a partir de un reordenamiento de la presencia de las distintas tendencias dentro de este liderazgo. Estos cambios son definitorios para la continuidad de la reforma y la inserción internacional de Vietnam. Entender las implicaciones de dicho evento es 
fundamental para valorar las posibilidades de Vietnam de mantenerse como una de las economías más dinámicas a escala internacional, y por tanto, en representar una de las alternativas que tienen tanto Colombia, como otros países de América Latina para sostener su tendencia al acercamiento comercial y financiero con Asia, dada la desaceleración que se pronostica para la economía china como parte del cambio en su modelo de crecimiento económico.

\section{El Partido Comunista y el contexto político vietnamita}

En el $6^{\circ}$ Congreso del PCV, efectuado en diciembre de 1986, se lanzó el "Doi Moi" (proceso de renovación económica en idioma vietnamita) y se inició un profundo proceso de reformas económicas, en la línea de la llamada "perestroika" que había promovido el entonces líder soviético Mijaíl Gorbachov, después de asumir el poder en 1985, y de las reformas de Deng Xiaoping, iniciadas en China a partir de 1978. Este proceso, significó la transformación de un modelo de economía centralmente dirigida hacia una "economía de mercado con orientación socialista”.

El "Doi Moi" se produjo en medio de un contexto político determinado por el fallecimiento, en ese mismo año, del entonces máximo dirigente del PCV Le Duan, considerado, entonces, como el principal representante de la llamada "línea dura" dentro del Partido, a favor del mantenimiento del llamado modelo de economía centralizada y del sistema político caracterizado por su fuerte ideologización "marxista-leninista". Por otra parte, Vietnam sufría el agravamiento de sus condiciones económicas ante la disminución de la ayuda económica proveniente de la Unión Soviética, debido a los problemas económicos que ya enfrentaba este país.

Desde el punto de vista económico, Vietnam ha vivido una profunda transformación. En los 30 años desde el inicio de las reformas, se ha permitido, y se protege constitucionalmente, la pro- 
piedad privada en empresas productivas y de servicios; el mercado ha reemplazado a la planificación centralizada como mecanismo de asignación de recursos; se ha desarrollado un cuerpo de leyes orientado a regular el funcionamiento del mercado, entre otras medidas de vital importancia. El país ha atraído una significativa inversión extranjera. Su nivel de inserción económica internacional ha mejorado ostensiblemente. Vietnam es una de las economías con mayor ritmo de crecimiento en el mundo y, a pesar de que aún existe un considerable nivel de pobreza, y de que en términos de ingreso per cápita, aún sigue distante de los niveles alcanzados por otras economías dinámicas de Asia, este indicador ha crecido considerablemente en los últimos años, reflejándose en un sustancial mejoramiento del nivel de vida de la población, en general.

Desde el punto de vista político, sin embargo, no se ha planteado una reforma sustancial. En 1992 se aprobó una nueva Constitución (reformada en 2013), la cual realizó cambios en la estructura del poder del Estado, pero no modificó aspectos esenciales del sistema político, tales como el liderazgo del PCV en el Estado y la sociedad, monopolizando el poder político. Aún se mantiene la ausencia de democracia en la elección de los diputados al parlamento y en la práctica, la elección de los principales funcionarios estatales por éste, no ocurre como resultado de la competencia de varios candidatos sino mediante la ratificación de quienes han sido designados por el Comité Central del PCV para ocupar dichas funciones. Por otra parte, la oposición sigue sin ser reconocida en el país y carece de legitimidad.

No obstante, se han producido algunos cambios positivos en la dirección del establecimiento de cierto equilibrio de poderes y una mayor separación de las funciones del Partido y del Gobierno. Se ha incrementado el rol de la Asamblea Nacional en el control de la actividad del gobierno. En 2012 se estableció el "voto de confianza" 
de los diputados sobre los principales funcionarios del Estado y el Gobierno, aunque solo es un voto de confianza alta, media y baja y en ningún caso, un voto de no confianza.

Adicionalmente, entre los 500 miembros de la $13^{\text {a }}$ Asamblea Nacional, 42 no son miembros del PCV, si bien ninguno de ellos se identifica como una oposición abierta al régimen.

Por otra parte, para evitar que los máximos dirigentes políticos ocuparan sus cargos por largos períodos de tiempo, hasta envejecer o fallecer en ellos, desde el $6^{\circ}$ Congreso del PCV se decidió, al igual que ya lo había hecho China, que ningún dirigente ocupe una de las máximas posiciones del Partido o el Estado por más de dos períodos (diez años). Esta disposición, significó el retiro de varios de los líderes que habían dirigido el país desde la década de los años 50's, entre ellos, Truong Chinh, Pham Van Dong, Le Duc Tho, Van Tien Dung, To Huu y Chu Hui Manh, entre otros, y ha traído como consecuencia la renovación paulatina del liderazgo vietnamita, a partir de entonces.

Así, desde esa época y hasta la más reciente elección, se han desempeñado cinco secretarios generales del PCV: Nguyen Van Linh (1986-91), Do Muoi (1991-97), Le Ka Phieu (1997-2001), Nguyen Duc Manh (2001-11) y el actual Nguyen Phu Trong (desde 2011). Por otra parte, desde el establecimiento de la nueva Constitución, la Asamblea Nacional ha elegido a cinco Presidentes del Estado: Le Duc Anh (1992-97), Tran Duc Luong (1997-2006), Nguyen Minh Triet (2006-11) y Truong Tan Sang (2011-16) y a Tran Dai Quang (desde 2016) y a cuatro Primeros Ministros: Vo Van Kiet (199297), Pham Van Kai (1997-2006), Nguyen Tan Dun (2006-16) y Nguyen Xuan Phuc (desde 2016).

Por lo general, los regímenes comunistas se han caracterizado por aparentar una unidad monolítica de sus equipos dirigentes. Sin embargo, esta situación ha estado muy lejos de ser real, sobre 
todo en los períodos de cambios profundos en los que se enfrentan corrientes reformistas con grupos conservadores, interesados en el mantenimiento del statu quo.

El liderazgo vietnamita, elegido en el $11^{\circ}$ Congreso del PCV y en la $13^{\text {a }}$ Legislatura de la Asamblea Nacional (ambos en 2011), mostró fracturas aparentes desde el mismo momento de su elección. Se podría incluso hablar de diferencias políticas entre las distintas facciones dentro del equipo dirigente, especialmente entre el Secretario General de PCV y el Presidente del Estado, de una parte, y el Primer Ministro, de otra. En ese momento, se comentaba en los corrillos políticos de Hanoi y de hecho fue publicado por diversos analistas de prensa que el Presidente Truong Tan San y el Primer Ministro Nguyen Tan Dun rivalizaron por esta última posición, la cual, de facto, tiene más poder real que la jefatura del Estado porque implica la dirección del gobierno y del poderoso entramado estatal que controla a las empresas estatales y a los gobiernos regionales, mientras que la Presidencia, tiene un poder principalmente ceremonial y de representación internacional.

Así, aparentemente, el Primer Ministro Dun era el político con mayor poder real en el grupo de dirigentes vietnamitas por su relación con las empresas estatales y con los gobiernos provinciales, y al mismo tiempo, era identificado con los altos niveles de corrupción que afectan al funcionamiento del aparato estatal en Vietnam, lo cual lo volvía vulnerable en la estructura del PCV.

Durante todo el período entre el $11^{\circ}$ y el $12^{\circ}$ congresos y la $13^{\mathrm{a}}$ y $14^{\mathrm{a}}$ Legislatura de la Asamblea Nacional, que se efectuó entre los últimos días de marzo y los primeros de abril de 2016, se hizo evidente el enfrentamiento entre las dos facciones dentro del liderazgo vietnamita. La que está encabezada por el secretario general del PCV Nguyen Phu Trong y el presidente Truong Tan Sang, se identifica como la facción conservadora "pro-china", interesada en 
mantener el curso de las reformas económicas pero fortaleciendo la labor ideológica del Partido y el control político que éste ejerce en la sociedad. La otra, liderada por el Primer Ministro Nguyen Tan Dung, es considerada como la facción "reformista", más orientada a una mejor relación con los países occidentales, especialmente Estados Unidos, y a profundizar las reformas económicas. Sin embargo, la realidad es que en ningún caso se identifica a un grupo dentro de la dirección de este país dispuesto a democratizar el sistema político. Por otra parte, resulta dudoso identificar una "facción pro-China" cuando es conocido el persistente conflicto entre Vietnam y China por las islas Spratley y Paracelso, el cual se ha agudizado recientemente, debido a la construcción, por parte de China, de islas artificiales en la zona de conflicto.

Por otra parte, en varias sesiones de la anterior legislatura de la Asamblea Nacional se produjeron cuestionamientos sobre la efectividad del gobierno en el manejo de la economía, y se produjeron críticas abiertas al Primer Ministro por cuestiones relacionadas con la economía. Esta situación se ha hecho evidente en los resultados de los votos de confianza en los que el Premier no salió muy bien librado, en comparación con otros dirigentes.

Entre marzo y mayo de 2012 se produjo uno de los más sonados juicios por corrupción, cuando varios ejecutivos de la empresa estatal Vietnam National Shipbuilding Industry Group (Vinashin) fueron juzgados por el Tribunal Popular debido a inversiones ineficientes que llevaron a pérdidas por 900 mil millones de dong (VND), aproximadamente 43 millones de USD al cambio de fines de 2016. Al mismo tiempo, los principales responsables de otra gran corporación estatal, Vietnam National Shipping Lines (Vinaline) fueron acusados de despilfarro y corrupción por pérdidas cercanas a los 81 millones de dólares en el solo ejercicio de 2009-2010 (Ver Bloomberg, 2013). 
Por otra parte, en agosto de 2012, se produjo el arresto de Nguyen Duc Kien, cofundador del Asia Commercial Bank y presidente del Club de Fútbol Hanoi ACB, de quien se decía tenía relaciones de amistad muy cercana con el Primer Ministro y su hija. Kien fue condenado a 30 años de cárcel por "violaciones económicas" en un sonado proceso en el que también fueron juzgados y condenados otros importantes ejecutivos de dicho banco (ver BBC, 2014).

En noviembre de ese mismo año, en una inusual sesión televisada del parlamento, el Primer Ministro fue directamente atacado por el diputado Duong Truc Quoc, y conminado a renunciar debido a errores en el manejo de la economía, así como consecuencia de los escándalos de corrupción descubiertos que involucraban a empresas públicas, cuyo desempeño no había sido adecuadamente monitoreado por el gobierno. Debido a lo extraño de la situación, muchos analistas concluyeron entonces que, muy probablemente, estas críticas fueron orquestadas desde el más alto nivel de dirección del PCV por rivalidades personales entre los máximos dirigentes del país (ver BBC, 2012).

Sus críticos, acusaban al Premier de haber construido una importante red de favores políticos y económicos a través de sus lazos con empresarios extranjeros y vietnamitas, de favorecer el enriquecimiento de su familia, de ser el responsable de la bancarrota de muchas empresas vietnamitas, así como de haber elevado excesivamente, la deuda pública vietnamita.

Sin embargo, en 2014, fue identificado como el principal impulsor de las reformas económicas, la privatización de empresas estatales, la incorporación al Acuerdo de Asociación Transpacífico (Trans-Pacific Partnership), así como ha sido el principal crítico de la exploración petrolera que China ha desarrollado en las islas Paracelso. Por estas razones, en el voto de confianza de ese año recibió un $64 \%$ de "alta confianza" y sólo un $14 \%$ de "baja confianza". Sin 
embargo, entre los principales líderes estatales del país (el secretario general del PCV no se somete a este tipo de voto), el Presidente Sang ha sido quien históricamente ha recibido el mayor número de votos de "alta confianza".

En el período previo al $12^{\circ}$ Congreso del PCV, muchos analistas especularon acerca de la renovación del liderazgo y algunos (Chánh, 2015) coincidían en que Nguyen Tan Dung sería el candidato más opcionado al cargo de Secretario General del PCV, debido a que no podría continuar como Primer Ministro por haber cumplido los dos períodos máximos en el cargo, tal y como establece la Constitución, y debido a que su popularidad en el país había aumentado en los últimos tiempos, y a que Nguyen Phu Trong probablemente no sería reelegido por su avanzada edad. Otros, (London, 2016) veían en Truong Tan Sang, como el más firme candidato a la Secretaría General del PCV, debido a su alta popularidad en el país (fue el miembro del Buró Político electo con la mayor cantidad de votos en el $11^{\circ}$ Congreso y ha sido el más votado con "alta confianza" en todas las votaciones llevadas a cabo en el parlamento del país). ${ }^{4}$

Sin embargo, los resultados de la elección del Comité Central y del Buró Político echaron por tierra las suposiciones de los analistas. El $12^{\circ}$ Congreso fortaleció la posición del Secretario General, Nguyen Phu Trong, quien no sólo fue reelegido en su cargo, sino que logró la exclusión de los otros cuatro principales dirigentes del país en el nuevo Buró Político, con lo que su posición como principal líder del país queda fortalecida y restablecida la tradición de que el secretario general, si bien no es ya el líder absoluto que antes era, al menos es el "primero entre iguales".

4 Ver Cang (2015) en relación a los cálculos políticos y expectativas que se existían antes del Congreso. 


\section{La conformación del nuevo liderazgo.}

En el nuevo Buró Político fueron ratificados sólo siete de los 16 miembros del anterior equipo. Los dirigentes ratificados fueron: el secretario general Nguyen Phu Trong, el ex-ministro de Seguridad y actual Presidente del Estado, general Tran Dai Quang, el anterior vice primer ministro y actual Primer Ministro, Nguyen Xuan Phuc, la vicepresidenta de la Asamblea Nacional de la $13^{\text {a }}$ Legislatura y recientemente elegida Presidenta de la Asamblea Nacional en la $14^{\mathrm{a}}$ Legislatura, Nguyen Thi Kim Ngang, el presidente del Frente Patriótico Nguyen Thien Nhan, el nuevo secretario ejecutivo del Secretariado Dinh The Huynh y la vicepresidenta de la Asamblea Nacional Tong Thi Phong. Los nuevos 12 miembros son: Vo Van Thuong, nuevo jefe de la Comisión Ideológica del CC; Tran Quoc Vuong, secretario de la Comisión Central de Inspección; Pham Binh Minh, viceprimer ministro y ministro de Relaciones Exteriores, Hoang Trung Hai, actual secretario del PCV en Hanoi; Dinh Le Thang, actual secretario del PCV en Ciudad Ho Chi Minh (Saigón); el general Ngo Xuan Lich, jefe del Departamento Político de las Fuerzas Armadas; To Lam, viceministro de Seguridad; Pham Minh Chinh, nuevo jefe de la Comisión de Organización del Comité Central (CC); Vuong Dinh He, jefe de la Comisión Económica del CC; Truong Thi Mai, vicepresidenta de la Comisión de Asuntos Sociales de la Asamblea Nacional de la $13^{\text {a }}$ Legislatura; Truong Hoa Binh, presidente de la Corte Suprema de Justicia; y Nguyen Van Binh, presidente del Banco Estatal de Vietnam.

El pasado 9 de febrero, se anunció la conformación del nuevo aparato operativo del Comité Central del PCV formado por el Secretariado y las Comisiones del Comité Central.

Dinh The Huynh (62), antiguo Jefe de la Comisión Ideológica del Comité Central ha pasado a la importante posición de secre- 
tario ejecutivo del Secretariado, lo cual lo convierte, de facto, en el segundo secretario del CC del PCV. Como parte de sus funciones tendrá la resposabiliidad de manejar los asuntos cotidianos del PCV. Si bien, esta posición no lo coloca necesariamente en la línea de sucesión, sin dudas lo convierte en uno de los cinco principales dirigentes del país, en términos de poder real, después del Secretario General, el Presidente, el Primer Ministro y la Presidenta de la Asamblea Nacional.

Vo Van Thuong, miembro del Buró Político y antiguo secretario del PCV en Ciudad Ho Chi Minh, pasó a la jefatura de la importante comisión de propaganda y educación del CC, la cual atiende los asuntos ideológicos. Mientras tanto, Truong Thi Mai quedó a cargo de la comisión de mobilización de masas. Hoang Trung Hai (actual viceprimer ministro) y Dinh La Thanh (actual ministro de Transporte), ambos elegidos nuevos miembros del Buró Político, fueron designados secretarios del PCV en las dos más importantes ciudades, Hanoi y Ho Chi Minh (antigua Saigón), respectivamente. Nguyen Van Nem, estará a cargo de la oficina administrativa del CC.

Así mismo, se conformó el Secretariado del Comité Central, integrado por el Secretario General Nguyen Phu Trong, el secretario ejecutivo, Dinh The Huynh; el jefe de la Comisión Central del Inspección, Tran Quoc Vuong; el jefe de la Comisión de Organización del CC Pham Minh Chinh, el jefe de la Comisión de Popularización y Educación del CC Vo Van Thuong; y la jefe de la comisión de Mobilización de Masas del CC Truong Thi Mai; así como por Luong Cuong, Nguyen Van Nem y Nguyen Hoa Binh.

Finalmente, entre fines de marzo y principios de abril de 2016, se efectuó la primera sesión de la $14^{\mathrm{a}}$ Legislatura de la Asamblea Nacional, en la cual se eligieron al Presidente y al Vicepresidente del Estado, al Primer Ministro, los Viceprimeros Ministros y los ministros del Gobierno y presidentes de los Comités Estatales, al 
Presidente (a) y Vicepresidentes de la Asamblea Nacional, así como a los Magistrados del Tribunal Supremo y al Fiscal General.

La nueva Asamblea Nacional eligió a los principales líderes del Estado siguiendo las recomendaciones del Comité Central del PCV, como ha ocurrido desde que se estableció el sistema comunista en este país. Como Presidente del Estado fue elegido el anterior ministro de Seguridad, general Tran Dai Quang (60), como Primer Ministro al hasta entonces viceprimer ministro Nguyen Xuan Phuc (62), y como Presidenta de la Asamblea Nacional a Nguyen Thi Kim Ngang (62), anterior vicepresidenta, la primera mujer en desempeñar esta función en este país. Todos ellos hacen parte del Buró Político del CC. Como nueva vicepresidenta del Estado fue elegida Dang Thi Ngoc Thinh (57), miembro del Comité Central del PCV. También fueron elgidos Nguyen Hoa Binh, miembro del secretariado del CC, como Presidente del Tribunal Supremo de Justicia y Le Minh Tri como Fiscal General.

El secretario general del PCV, Nguyen Phu Trong, ha tenido una larga carrera en el aparato político del Partido y en diversas posiciones estatales. Nació en el seno de una familia de campesinos pobres en el distrito de Dong Anh, en la zona rural de Hanoi, pero estudió Filología en la Universidad Nacional de Vietnam. Durante muchos años fue editor de la revista teórica del PCV, "Tap chi Congg San” (Revista Comunista). Obtuvo un doctorado en Historia en la Academia de Ciencias Sociales de la URSS y posteriormente se convirtió en uno de los principales teóricos del PCV, ingresando en el Consejo Teórico del CC. Ha sido miembro del CC del PCV desde 1994 y del Buró Político desde 1997. Ha sido diputado en la Asamblea Nacional desde 2002. Entre 2000 y 2006 fue secretario del PCV en Hanoi. Entre 2006 y 2011 fue Presidente de la Asamblea Nacional de Vietnam y desde 2011 es el Secretario General del PCV. 
El Presidente Tran Dai Quang, estudió en la Escuela de la Policía Popular y en la Escuela de Lenguas Extranjeras del Departamento de Asuntos Culturales del Ministerio de Seguridad Pública. Durante varios años fue oficial del departamento de Protección Política del dicho ministerio. Toda su carrera se ha desarrollado en este ministerio en el que ha desempeñado funciones directivas. En 2003 fue ascendido a General Mayor, en 2007 a Teniente General y en 2011 a Coronel General. Entre 2006 y 2011 fue viceministro de seguridad Pública y entre 2011 y 2016 se desmepeñó como Ministro. Estudió en la Universidad de Leyes de Hanoi.

El nuevo Primer Ministro, Nguyen Xuan Phuc, se graduó en Economía en la Universidad Nacional de Economía de Hanoi y en gestión administrativa en la Academia Administrativa de Vietnam y estudió Administración Económica en la Universidad Nacional de Singapur. Desempeñó diversas funciones administrativas y de gestión económica en su provincia natal de Quang Nam, en la cual fue vicepresidente del Comité Popular Provincial. Entre 1994 y 2002 fue presidente de dicho comité. Entre 2007 y 2011 fue ministro del Gobierno y entre 2011 y 2016 se desempeñó como Primer Viceprimer Ministro.

La nueva Presidenta de la Asamblea Nacional, Nguyen Thi Kim Ngan, estudió Ciencias Políticas y obtuvo un master en Administración Financiera y Crediticia. Fue ministra de Trabajo, Asuntos Sociales e Inválidos. Entre 2011 y 2016 fue vicepresidenta de la Asamblea Nacional y desde 2011 es miembro del Buró Político del CC del PCV.

En el nuevo Gabinete todos los viceprimeros ministros y ministros son miembros del Comité Central y cinco de ellos hacen parte del Buró Político, lo cual demuestra el nivel de relación entre las estructuras del Partido y del Gobierno. De los cuatro Viceprimeros ministros, tres son miembros del Buró Político, lo que hace suponer 
que alguno de ellos sería el próximo jefe del Gobierno. Los nuevos viceprimeros ministros son: Pham Binh Minh (ministro de Relaciones Exteriores), Truong Hoa Binh, Vuong Din Hue (jefe de la Comisión Económica del CC del PCV), y Trinh Dinh Dung (miembro del CC del PCV). Otros miembros del Buró Político designados para el nuevo gobierno son el nuevo ministro de Defensa, general Ngo Xuan Lich y el nuevo ministro de Seguridad Pública, general To Lam, lo cual destaca, además, la importancia de los organismos castrenses en la estructura del poder político del país.

\section{Comentarios finales}

A falta de la información suficiente sobre las características y posibles inclinaciones en la dicotomía reformistas-conservadores que suele caracterizar la dirigencia de países totalitarios comunistas, se pueden realizar algunas consideraciones generales.

Se ha fortalecido, al menos temporalmente, la posición del Secretario General Nguyen Phu Trong, quien es el miembro de más edad del nuevo Buró Político, y al lograr la salida de los otros cuatro principales dirigentes de la anterior legislatura, refuerza su senioridad dentro del nuevo equipo dirigente. Es posible, sin embargo, que pudiera ser reemplazado antes de concluir su nuevo mandato de cinco años, en razón de su edad, cuando se hubiera consolidado este nuevo liderazgo. Trong es identificado como el principal líder "conservador", lo cual, sin embargo, no debe sugerir que sea partidario de revertir las reformas, sino más bien en la línea de reforzar el control político e ideológico del PCV sobre la vida cotidiana del país e incluso, sobre la administración estatal, debido a la erosión de prestigio sufrida por las sistemáticas críticas a la corrupción que aparecen en los periódicos nacionales y que son abiertamente mencionadas tanto por inversionistas extranjeros como por empresarios nacionales. 
El predominio de esa línea "conservadora" se ratifica por el ascenso a la segunda posición, dentro del PCV, del general Tran Dai Quang, elegido recientemente para el cargo de Presidente del Estado. En algún momento anterior al Congreso se le consideró como un posible un candidato de compromiso a la Secretaría General del PCV, ante la eventualidad de que todos los principales dirigentes fueran reemplazados. Desde su puesto como anterior ministro de Seguridad, Quang ha sido considerado muy poco tolerante respecto a los activistas vietnamitas en favor de la democracia. Como Presidente, podrá adquirir experiencia en los asuntos de política internacional, los que sumará a su experiencia en las cuestiones de seguridad y tendrá la perspectiva global de la política doméstica e internacional, incluyendo la política económica y esto, junto a su edad, lo convertiría en un fuerte candidato futuro a la secretaría general del Partido.

Otro elemento que fortalece la idea de un leve giro hacia una tendencia "conservadora" es la designación de Dinh The Huynh, como secretario ejecutivo del secretariado, puesto que su anterior cargo era el de Jefe de la Comisión Ideológica y de Educación del CC, que suele ser el guardián de la ortodoxia ideológica en el Partido.

Habrá que esperar cuál será la posición del nuevo Primer Ministro en relación con las reformas necesarias para la economía vietnamita, lo cual queda bajo su directa responsabilidad como jefe del gabinete. El mantenimiento de los altos ritmos de crecimiento económico y el mejoramiento del nivel de vida de la población son elementos clave en el mantenimiento del actual "contrato social".

En el nuevo equipo se establece una suerte de cierto desequilibrio de procedencia entre las tres grandes zonas geográficas en las que se divide el país (norte, centro y sur), lo cual suele resultar importante, debido a las diferencias históricas entre las mismas. En- 
tre los cinco principales dirigentes del país, tres proceden del norte: Nguyen Phu Trong (zona rural de Hanoi), Tran Dai Quang (Ninh Binh) y Dinh The Huynh (Nam Dinh), mientras que el Primer Ministro Nguyen Xuan Phuc procede de la provincia de Quang Nam en el centro y la Presidenta de la Asamblea Nacional, Nguyen Thi Kim Ngang, procede de la provincia de Ben Tré en el sur. En anteriores equipos dirigentes se había tratado de mantener un cierto equilibrio entre norte, centro y sur, al menos en las tres principales posiciones del país.

Los cambios en la cúpula dirigente del país muestran, sin embargo, una relativa continuidad en el objetivo de mentener el rumbo de la creciente inserción internacional del país en la economía mundial y en el sistema de relaciones internacionales. El dinamismo de su economía, una de las de más rápido crecimiento en el mundo, y su creciente participación en el comercio internacional y en las corrientes de inversión extranjera directa, así como los crecientes, aunque aun discretos, vínculos comerciales con Colombia, deben llevar tanto a las autoridades del país como a su sector empresarial a considerar a este país como de gran interés estratégico en lo que se refiere a sus relaciones con el continente asiático. Como un país en el que el Estado tiene una gran influencia en el comportamiento de la economía, el conocimiento de las variables políticas resulta de gran importancia para el trazado de la política exterior de Colombia, así como para la estrategia empresarial en el desarrollo de las relaciones bilaterales.

\section{Bibliografía.}

BBC (2012), Vietnam Prime Minister Nguyen Tan Dung urged to resign. Recuperado de http://www.bbc.com/news/world-asia-20322830

BBC (2014), Vietnam soccer tycoon Nguyen Duc Kien gets 30 years. Recuperado de http:// www.bbc.com/news/world-asia-27758693 
Bloomberg (2013), Vietnam's Corruption Crackdown Shown in Vinalines Trial. Recuperado de https://www.bloomberg.com/news/articles/2013-12-12/vietnam-s-corruption-crackdown-shown-in-vinalines-trial

Cang Công Phan (2015) Vietnam After 2016: Who Will Lead?. Recuperado de http://thedipIomat.com/2015/07/vietnam-after-2016-who-will-lead/

Chu, T. T. H. and Dickie, P. M. (2006) "Economic transition in Viet Nam: DoiMoi to WTO", Public Policy Training Program (PPTP) Studies Series 1, Asian Development Bank

EFE (2016), El Partido Comunista de Vietnam debate el liderazgo del país para los próximos años. Vietnam. Recuperado de http://www.efe.com/efe/america/portada/ el-partido-comunista-de-vietnam-debate-liderazgo-del-pais-para-los-proximos-anos/20000064-2815567

London, Jhonathan (2016) Vietnam's Leadership Succession Struggle. http:// thediplomat.com/2016/01/vietnams-leadership-succession-struggle/ SELA. (2016). Análisis de las relaciones económicas de América Latina y el Caribe con la Asoción de Naciones del Sudeste Asiático (ASEAN). Sistema Económico Latinoamericano y del Caribe .

Trade Map (2015) www.trademap.org

UNCTAD (2015) UNCTADStat. www.unctad.org

Vidal Alejandro, P., Peláez, J. T., \& De Miranda, M. (2015). Restricciones De Balanza De Pagos En Colombia. Efectos De La Apertura Internacional Y El Aumento Del Comercio Con Asia. Documentos de Trabajo No. 3, Facultad de Ciencias Económicas y Administrativas, Universidad Javeriana Cali.

Vidal, Pavel y Lina Rodriguez (2015). Vulnerabilidades Del Crecimiento Colombiano a La Desaceleración de la Economía China, en de Miranda, M. y Pelaez, J.T (2015) Las Relaciones Económicas entre América Latina y Asia, Sello Editorial Javeriano. 